\title{
Clinical case of a male patient with oral ulcerative lesions due to eppstein-barr virus and cytomegalovirus
}

\author{
Assya Krasteva ${ }^{1}$, Yanitsa Istatkova ${ }^{1}$, Anelia \\ Gozeva ${ }^{2}$,
}

1. Department of Imaging and Oral Diagnostics, Faculty of Dental Medicine, Medical University - Sofia, Bulgaria;

2. Department of Virusology, Military Hospital - Sofia, Bulgaria;

\begin{abstract}
Epstein-Barr virus and Cytomegalovirus have different oral manifestations which could be presented in the oral cavity most commonly as non-specific oral ulcerations and lymphadenopathy. A lot of people acquire primary infection with Cytomegalovirus and if they later become immunosuppressed, such as human immunodeficiency virus (HIV), Cytomegalovirus is likely to become reactivated.
\end{abstract}

We present a clinical case of a young man with multiple painful oral ulcerations with duration more than several years. The patient applied different types of local creams without significant results. After the clinical examination our recommendation was to investigate serum levels of EBV- IgG and CRP- IgG and HIV-1 and -2, complete blood count with differential (CBC), folic acid, CRP.

The laboratory results revealed positive IgG antibodies against CMV and EBV, folic acid deficiency.

Keywords: CRP, CMV, EBV, folic acid, oral ulcerations. 


\section{Background}

Cytomegalovirus (CMV) is responsible for a significant percentage of asymptomatic viral infections worldwide. It is important that any cell or organ may be infected (1).

Like all herpesviruses, EBV establishes a life-long, persistent infection of its host and in the majority of humans without causing a disease. JA Thomas and co-workers published that there are reports in the literature about EBV infection in normal T- cells and neoplastic T-cell diseases. Generally EBV is held to infect B- cells and epithelial cells (2).

The effects of human CMV on cellular functions, which may be associated with the malignant phenotype, include the expression of oncogenes and transcriptional activation of growth factors and interleukin synthesis.

In the oral cavity CMV may be presented as a single, large necrotic painful ulcer and less often as multiple ulcers, which last for weeks or months and any site may be involved. Up to one-third of such ulcers are co-infected with other viruses of the herpes family, especially herpes simplex virus (HSV) and varicella zoster virus (VZV). There have been occasional reports of mandibular osteomyelitis and tooth exfoliation associated with CMV and VZV infection. Some authors have reported CMV oral lesions in HIV-infected patients and emphasize that CMV mucosal ulceration may be the initial manifestation of AIDS (2).

Both EBV and CMV could be transmitted with body fluids (saliva, breast milk, respiratory secretions), by sexual contact and blood transfusion; additionally, EBV could be transmitted by sharing food, drinks or eating utensils with an infected person and CMV- during delivery and after organ transplantation (2).

EBV could cause infectious mononucleosis, oral hairy leucoplakia, Burkitt lymphoma, Hodgkin lymphoma, nasopharyngeal carcinoma, etc. The primary infection with CMV may be asymptomatic or cause an infectious mononucleosis- like disease (2).

\section{Co-infection with Cytomegalovirus and Epstein-Barr virus (EBV)}

There are reports in the medical literature concerning co- infection with CMV and EBV.

D. Olson and co-workers have found elevated IgM titers to both CMV and EBV in young women (3). Both near- simultaneous infections and temporally remote sequential infections with acute CMV triggering an immunoreactivation of EBV are reported in the literature. Recognizing that co-infection with these viruses may modulate the clinical course of disease is important (1).

Krasteva et co-workers have investigated serum levels of IgM and IgG to Cytomegalovirus and EpsteinBarr virus in 116 consecutive dental patients with non- specific oral lesions. The authors have detected elevated IgM antibodies to CMV in $2.8 \%$. $6 \%$ of patients have been positive for VCA- IgM antibodies. Positive VCA-IgG have been detected in almost $97 \%$ of all tested dental patients and this has revealed that these people had a current or recent EBV infection and may have reactivation of EBV (4). 
Folic acid (vitamin B9) is one of the vitamins of group B. It is a water-soluble vitamin; it is absorbed from the small intestine and stored in the body in insignificant quantities. $7 \mathrm{mg}$ are stored in the liver and other tissues but they could protect from a deficiency only for a couple of months $(4,5)$. Folate deficiency may lead to diarrhea, depression, confusion, megaloblastic anemia and fetal neural tube defects and brain defects (during pregnancy). The most common folic acid deficiency cause is a low daily intake from foods (lack of fresh green vegetables, legumes), and prolonged storage and cooking could cause 50\% to 95\% loss of folate. Other factors that may cause folic acid deficiency are alcoholism, malabsorption, pregnancy, lactation, elderly age, bowel diseases (Crohn's disease, ulcerative colitis, celiac disease) and certain drugs (tetracyclines, trimetoprim, cytostatic drugs, metothrexate, antiacidic drugs- omeprazole, pantoprazole, anti- epileptic drugs- phenitoin, non-steroidal anti- inflammatory drugs (NSAID)). Folic acid deficiency may not be so obvious in the early stages, it may take up to four months before any signs and symptoms are manifested $(4,5)$.

The orofacial manifestations of a folate deficiency include: angular cheilitis, mucositis, stomatitis, symptoms of burning mouth syndrome, glossodynia, glossitis with redden pick and/ or lateral borders, edematous pick of the tongue, pale tongue, depapilated and atrophic glossitis, aphtous ulcers, high risk of oral candidiasis, inflammated gingiva, ulcerative gingivitis $(4,5)$.

\section{Case description}

We present a case of 25 years- old male patient who came to the Department of Imaging and Oral Diagnostics, Faculty of Dental Medicine, Medical University- Sofia complaining of painful recurrent aphtous ulcers with duration more than several years. The patient applied different types of local creams without significant results.

The patient underwent diagnostic protocol which included:

1. Clinical examination - dental status, palpation of lymph nodes, percussion;

2. Taking a series of photos for his medical records:

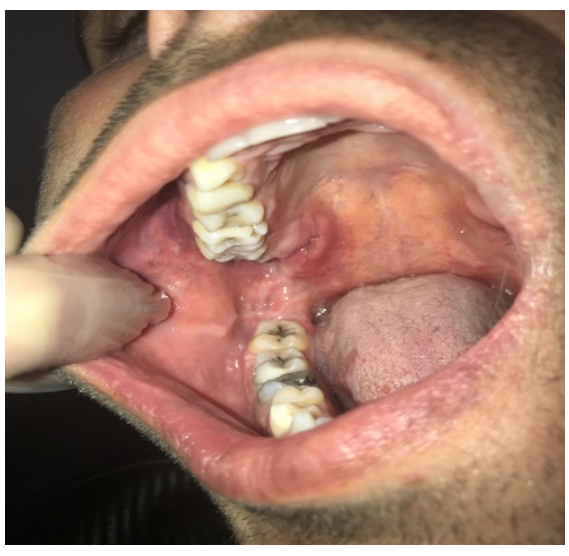

Figure 1 Painful oral ulceration on the right palatal mucosa

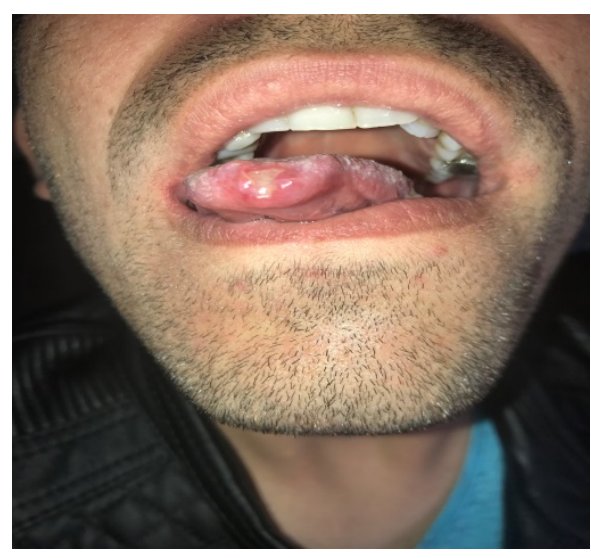

Figure 2 Large oral ulcer on the pick of the tongue 


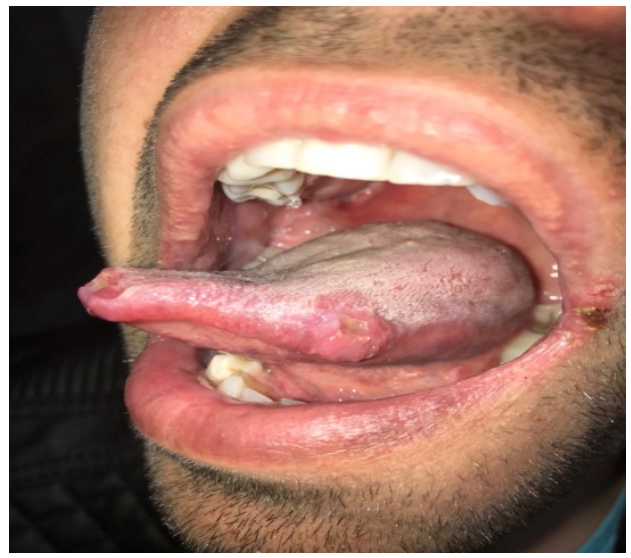

Figure 3 Indurative oral ulcer on the left lateral border of the tongue

3. Complete blood count with differential (CBC), including additionally levels of CRP, vitamin B9 (folic acid), vitamin B12, erythocyte sedimentation level (ESR), EBV, CMV, HIV.

\section{Results}

During the clinical examination was observed that the patient has multiple ulcers located on the soft palate, pick of the tongue and the left lateral surface of the tongue. The lesions were well demarcated, erythematous and with sharp borders; the consistency was soft. They were covered with yellowish pseudo-membranous plaque. The tissue around the lesions was edematous and erythematous. The patient claimed that these lesions were very painful on palpation.

\section{Laboratory tests - results:}

$\mathrm{CRP}=10.8 \mathrm{mg} / \mathrm{l}$ (normal value $=<5 \mathrm{mg} / \mathrm{l})$,

Folic acid $=3.94 \mathrm{nmol} / \mathrm{l}$ (normal values= 8.8- 60.8nmol/l);

CMV- IgG= $153.8 \mathrm{U} / \mathrm{ml}$ serum/ECLIA (normal values $=<4$ ),

EBV- VCA IgG= $154 \mathrm{U} / \mathrm{ml}$ serum/CLIA (normal values $=<20$ ),

Anti- HIV 1+2 serum/ ELISA= reactive (normal value= negative).

\section{Dental recommendations:}

The patient was sent to e referent laboratory for confirmation of the results. The second samples clarified that the patient was negative for anti-HIV $1+2$.

\section{He was given a therapy with:}

1. "BioGaia Prodentis"- $2 \times 1$ tablet daily for 3 months;

2. "Geritamin"- 1 tablet daily for 3 months;

3. "Milgamma N"- $3 \times 1$ tablet for 3 months; 
4. "Aloclair gel" for local treatment of aphtous ulcers;

5. "Xyzal" tablets of $5 \mathrm{mg}, 1$ tablet in the evening;

6. "Isoprinosine" tablets of 500mg, $2 \times 2$ tablets daily;

7. Multivitamins Vitamin C;

8. Removing of all amalgam obturations;

9. Giving recommendations for keeping proper personal oral hygiene, new toothbrush with soft bristles;

10. Dental treatment of all carieses.

\section{Discussion}

The oral ulcers are among the most common disorders of the oral mucosa. The patient may come to the dentist observation with the unique manifestation of the pain symptom caused by the ulcer it-self. Oral ulcers may be due to Coeliac disease or Crohn's disease. Some Viral infections, particularly HSV1, HSV2, and, more rarely, EBV and CMV, etc. In the presence of a state of immunodeficiency or lack of sufficient levels of folic acid, may be induced some superficial oral ulcers, often associated with labial manifestations $(6,7)$.

In immunocompromised patients, oral ulcerations are common and have a wide spectrum of causes, including herpesvirus infection (8).

Cytomegalovirus (CMV) may give rise to large, chronic ulcers of the oral mucosa or gingiva. These CMVrelated ulcers occur exclusively under conditions of significant immunodeficiency, notably severe HIV disease. The diagnosis of such ulcers is difficult and is often only confirmed by resolution of ulcers with ganciclovir therapy (7). Schubert and co-workers in a literature review have discussed the prevalence of oral ulcers in immunocompromised patients positive to cytomegalovirus (9).

Mainville and co-workers have reported on a case in which an oral ulcer has been simultaneously infected by herpes simplex (HSV), cytomegalovirus (CMV), and Epstein-Barr virus (EBV) in a kidney-pancreas transplanted recipient. The authors have suspected that the pathogenesis of co-infected ulcers remains unknown, a synergistic effect is possible (8).

Different studies have presented patients with oral manifestation, due to association between CMV, EBV and HIV $(10,11,12)$, but our patient was negative to HIV.

Mario Dioguardi et co-workers have presented a 59-year-old woman with complaints of a painful roundish ulcer, covered by fibrinous exudate, lasting for 1 month. The authors have performed immunohistochemical coloration with monoclonal "anti-CMV" antibodies to detect viral proteins and the result was positive. The ulcer has improved rapidly following the initiation of ganciclovir treatment (6). 


\section{CMV and EBV treatment}

The antiviral medications against CMV include the following:

- Ganciclovir (Cytovene) is the first antiviral medication given intravenously. Drug side effects include fever, rash, diarrhea, anemia, and low white blood cell and platelet counts (13).

- Valganciclovir (Valcyte) is an oral medication that is activated to ganciclovir in the body and is as effective as intravenous ganciclovir in milder cases (13).

- Foscarnet (Foscavir) is active against CMV by a different mechanism than ganciclovir. Drug side effects include nephrotoxicity, seizures due to an imbalance of minerals and electrolytes (13).

- Cidofovir (Vistide) is an alternative therapy for patients who have failed treatment with ganciclovir and foscarnet. It has less nephrotoxicity effect. It is used mainly for the treatment of CMV in patients with acquired immune deficiency syndrome (AIDS) (13).

Efficacy of cidofovir is similar to that of ganciclovir or foscarnet. Significant adverse effects, including renal failure, limit its use. Cidofovir may cause iritis or ocular hypotony (intraocular pressure $\leq 5 \mathrm{~mm} \mathrm{Hg}$ ). The potential for nephrotoxicity can be reduced by giving probenecid and prehydration with each dose. However, the adverse effects of probenecid, including rash, headache, and fever, may be significant enough to prevent its use (14).

- CMV immune globulin contains antibodies (proteins) that are specific to CMV. It may be prescribed to prevent CMV infection in high-risk lung transplant patients when given in addition to ganciclovir. It is also used with ganciclovir to treat CMV pneumonia (13).

We started our treatment with folic acid and Isoprinosine. After 1 month the patient came to reevaluation, but the treatment was not so successful. The patient was referred to be hospitalized and to be chosen another antiviral drug.

\section{Conclusion}

Patients with oral ulceration can be challenging to diagnose and manage. Patients with signs or symptoms of oral ulcers are sometimes referred to gastroenterology clinics; and less commonly to the dentists. Considering the wide variety of pathological conditions that can lead to oral ulcerations, it is evident the importance of a correct diagnosis for the appropriate treatment. In unspecific ulcerations it must be suspected viral etiology - usually HSV, EBV, CMV and HIV. Our patient has co-infection between CMV and EBV. Our initial treatment was not so successful and we made a re-evaluation of the treatment plan.

\section{References}

1. Krasteva A, Ivanova A, Kisselova A. Prevalence of Epstein-Barr virus and cytomegalovirus in Bulgarian dental patients; Medinform 2016; 3:2:463-471.

2. Krasteva A. Epstein-Barr virus and Cytomegalovirus - two herpes viruses with oral manifestations. J of IMAB 2013; 19:4: 359-362. 
3. Olson D, Huntington M. Co-infection with cytomegalovirus and Epstein-Barr virus in mononucleosis: case report and review of literature. South Dakota Medicine: the Journal of the South Dakota State Medical Association 2009, 62 :9: 349, 351-353.

4. Krasteva A. Anemia and nutrition deficiency in dental practice, Варненски медицински форум, т. 5, 2016, брой 2, 197- 203.

5. Krasteva A., Kisselova A., Krastev Z. Folic acid and vitamin B12 levels in bulgarian patients with burning mouth syndrome. J of IMAB 2013 Jul-Dec;19:4:422-425.

6. Dioguardi M, TroianoG, Russo L, et al. Occult co-infection in the oral cavity with cytomegalovirus during immuno-suppression. J Transl Sci, 2015 Volume 1:2: 26-28.

7. Leão JC, Gomes VB, Porter S. Ulcerative lesions of the mouth: an update for the general medical practitioner.CLINICS 2007;62:6:769-780.

8. Mainville GN, Marsh WL, Allen CM. Oral ulceration associated with concurrent herpes simplex virus, cytomegalovirus, and Epstein-Barr virus infection in an immunocompromised patient. Oral Surg Oral Med Oral Pathol Oral Radiol. 2015 Jun;119:6:306-314.

9. Schubert $\mathrm{MM}$, Epstein JB, Lloid $\mathrm{ME}$, et al. Oral infections due to cytomegalovirus in immunocompromised patients. J Oral Pathol Med 1993; 22: 268-273.

10. Firth NA, Rich AM, Reade PC. Oral mucosal ulceration due to cytomegalovirus associated with human immunodeficiency virus infection. Case report and brief review. Aust Dent J 1994; 39: 273275.

11. Syrjanen S, Leimola-Virtanen R, Schmidt-Westhausen A, et al. Oral ulcers in AIDS patients frequently associated with cytomegalovirus (CMV) and Epstein-Barr virus (EBV) infections. J Oral Pathol Med 1996;28: 204-209.

12. Bunn B, van Heerden W. EBV-positive mucocutaneous ulcer of the oral cavity associated with HIVIAIDS. Oral Surg Oral Med Oral Pathol Oral Radiol 2015.

13. Gonzalez S. Cytomegalovirus Infection. https://www.medicinenet.com/cytomegalovirus cmv/article.htm\#cytomegalovirus cmv i nfection facts

14. Kaye K. Cytomegalovirus (CMV) Infection. (Cytomegalic Inclusion Disease) https://www.msdmanuals.com/professional/infectious-diseases/herpesviruses/cytomegaloviruscmv-infection

\section{Corresponding author:}

Dr. Yanitsa Istatkova

Department of Imaging and Oral Diagnostics,

Faculty of Dental medicine,

Medical University - Sofia

Bul. Sv. Georgi Sofiiski,

Sofia 1000, Bulgaria,

email: q_a_istatkova@abv.bg 